\title{
Open Bite Malocclusion as Potential Predisposing Risk Factor to Promote Periodontal Disease
}

\author{
Jose Ricardo Kina ${ }^{1 *}$, Juliana Kina ${ }^{2}$, Monica Kina ${ }^{3}$ and Eunice Fumico Umeda Kina ${ }^{4}$ \\ ${ }_{1}^{1}$ Department of Periodontology, FOA-UNESP, Universidade Estadual Paulista Julio de Mesquita Filho Araçatuba, São Paulo, Brazil \\ ${ }^{2}$ Private Orthodontist, Araçatuba, São Paulo, Brazil \\ ${ }^{3}$ Department of Restorative Dentistry, UNICASTELO-Fernandopolis Dental School, São Paulo, Brazil \\ ${ }^{4}$ Private Practice, Araçatuba, São Paulo, Brazil
}

*Corresponding author: Jose Ricardo Ricardo kina, Universidade Estadual Paulista Julio de Mesquita Filho, Araçatuba, São Paulo, Brazil, Tel: 551836226769; E-mail: kinajr@hotmail.com

Received date: July 13, 2016; Accepted date: Aug 22, 2016; Published date: Aug 26, 2016

Copyright: (c) 2016 Kina JR, et al. This is an open-access article distributed under the terms of the Creative Commons Attribution License, which permits unrestricted use, distribution, and reproduction in any medium, provided the original author and source are credited.

Citation: Kina JR, Kina J, Kina M, et al. Open Bite Malocclusion as Potential Predisposing Risk Factor to Promote Periodontal Disease. J Orthod Endod. 2016, 2:3.

\section{Abstract}

Periodontal disease is caused by a necessary association of bacteria and other risk factors. The open bite malocclusion determined by mouth breathing condition could be one of the risk factors that aid bacteria to induce a local periodontal disease in the anterior teeth by a mechanism that was analyzed in this article. One hundred and twenty adult patients of both genders presenting an open bite malocclusion due to a mouth breathing condition were randomly selected and analyzed to establish a possible correlation with periodontal disease in the anterior teeth. Ninety-five percent of the patients studied were diagnosed with a periodontal disease in the anterior teeth. The results obtained demonstrated that periodontal disease in the anterior teeth is prevalent in patients presenting open bite malocclusion.

Keywords: Bacteria; Etiology; Open bite malocclusion; Periodontal diseases

\section{Introduction}

Open bite malocclusion is a divergence in the vertical dimension between the mandibular and maxillary anterior dental arches; it is characterized by a loss of vertical dental edge contact in opposing anterior segments of the teeth [1]. The etiologic factors that lead the individual to develop an open bite malocclusion relationship are an interplay of various different etiologic factors, which are hereditary and environmental in nature [2-7]. The periodontal disease etiology is also multifactorial and bacterial plaque plays an essential role in inducing the disease $[8,9]$. Although bacteria are critical to developing a periodontal disease, bacteria alone are insufficient to provoke a periodontal disease; a susceptible host is necessary, and host susceptibility as local and/or general risk factors are vital determinants of disease status [10-15]. The risk factor may be an intrinsic feature related with an amplified rate of a subsequently occuring disease, but it does not inevitably induce the disease. In periodontal disease, the risk factors may be defined as behavioral in nature, or environmental factors such as developmental or acquired deformities and conditions that affect the periodontium. They may also be local and systemic factors, which may be responsible for providing fragility in a determinate tooth or teeth and adjacent periodontal tissues or an indispensable environment for bacterial colonization or interference in the inflammatory process $[14,15]$. An accurate diagnosis is often critical to developing a well designed and appropriate treatment plan that when implemented leads to a resolution of the patient's periodontal disease infection. Thus the principal aim of this study is to analyze and discuss how, in some individuals, the open bite malocclusion may contribute, interacting as an etiologic predisposing risk factor to induce bacteria to provoke a local periodontal disease.

\section{Materials and Methods}

The strategy of this study was to analyze a group of randomly selected individuals who were diagnosed with untreated open bite malocclusion due to a mouth breathing condition to try to establish a possible correlation with a localized periodontal disease in the anterior teeth. The study sample comprised of 120 adult individuals of both genders, 50 women and 70 men, ages ranging from 18 to 55-years old, selected randomly from a private dental office located in Araçatuba-São Paulo, Brazil, by clinical and radiographic methods. It was made clear to all potential subjects that participation was voluntary. All subjects were made aware of the purpose of our research and the methodology, and they signed an informed consent for participation in the study. 
Inclusion criteria: Selection criteria comprised of ages above 18-years and a mean number of 26 natural teeth, since missing teeth might have interfered with the results. The sample included individuals presenting open bite malocclusion diagnosed via clinical and radiographic means by an experienced orthodontist (J.K.). To diagnose periodontal disease, the parameter applied waspresence of periodontal attachment loss. A millimeter probe marked up to $15 \mathrm{~mm}$ (HuFriedy model Color Coded Probe, code CP-15UNC-PCPUNC15, USA), was applied for assessment of all teeth of each individual by a single examiner (J.R.K.) Four surfaces were examined in each tooth: Mesial, buccal, distal and lingual, and the bone loss in the area was confirmed by radiographic means. To register the periodontal attachment loss, a linear measure was obtained from the cemento-enamel junction (CEJ) until the bottom of the gingival sulcus or periodontal pocket in each tooth. This was registered whenever there was equal to or more than $4 \mathrm{~mm}$ of periodontal attachment loss at least on one surface of each evaluated tooth [13]. Exclusion Criteria: Subjects who had periodontal treatment in the previous 12 months or any medical condition that would require antibiotic prophylaxis for routine dental procedures. Individuals who had taken antibiotics in the previous 3 months or who were pregnant were also excluded from this study.

\section{Results}

Periodontal diseases were diagnosed in anterior teeth in 114 individuals, corresponding to $95 \%$ of the total sample (Figures 1A-1E). All of the 114 diseased individuals displayed at least one anterior tooth presenting sequelae of periodontal disease as periodontal attachment loss equal to or more than $4 \mathrm{~mm}$ measured from the CEJ. Regarding gender aspect, periodontal attachment loss was diagnosed in 46 women and 68 men in the anterior region. Six individuals presented some parameters of the periodontal attachment loss but presented gingivitis in the anterior teeth (Figure 2). Among the 114 individuals with a periodontal disease in the anterior teeth, all of them presented a worse periodontal condition in the anterior teeth, 108 presented some attachment loss in other areas, 6 presented some periodontal attachment loss in the posterior teeth and 39 were missing at least 1 anterior tooth. The most frequently affected teeth by periodontal disease were 31 and 41, mainly by gingival recession or the teeth 31 and/or 41 were absent. All the individuals without any periodontal diseases were related to the subjects aged between $18-19$ years old.

\section{Discussion}

Etiology encompasses the sum knowledge relating to the origin of a disease. The concepts of the origin of inflammatory gingival and periodontal diseases are an intricate interaction among bacteria and other risk factors, such as local, environmental, emotional, and systemic factors [10-15]. Although bacteria are undoubtedly etiologically associated with periodontal diseases, efforts to diagnose mainly the other risk factors may be essential to treating periodontal disease $[14,15]$
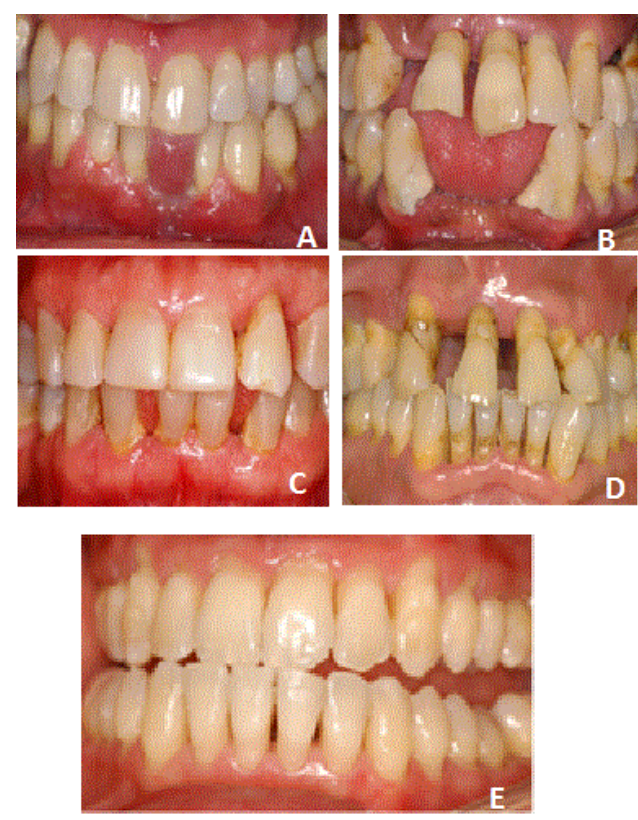

Figure 1: Diagnosis of Periodontal diseases in anterior teeth $(A-E)$.

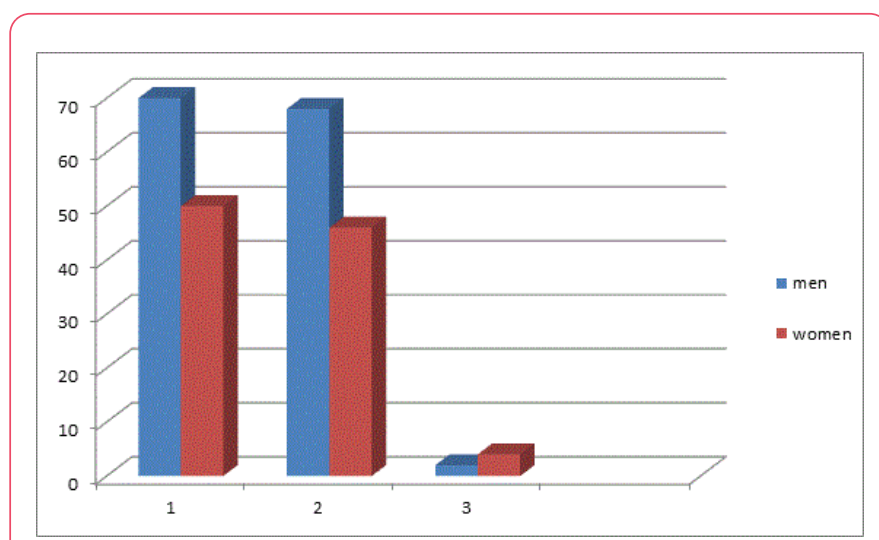

Figure 2: Attachment loss in anterior teeth according sex [N=120; 1: Individuals; 2 : Individuals presenting attachment loss in anterior teeth; 3: Individuals without attachment loss in anterior teeth].

The open bite malocclusion, considered a deviation in the vertical relationship of the maxillar and mandibular dental arches and with prevalence around $3.5 \%$ to $16.5 \%$ among several races in the world, could be in a determined moment one of these other risk factors which aid bacteriainducing local periodontal disease [14]. The main etiologic factors that lead an individual to develop an open bite malocclusion are deleterious oral habits such as fin ger sucking or lip and tongue position and the mouth breathing condition $[1,3,4]$. The mouth breathing condition may influence the inactive posture of the lips, teeth location, and the functional tongue movement, and may provoke an open vertical dimension between the incisal edges of the maxillary and 
mandibular anterior teeth [5-7]. The tooth erupts within its arch into a certain position according to the two horizontally opposed forces which characterize a determinant of the occlusion described as a neutral zone. The inward pressure generated by the three buccinator muscle bands against the outward pressure of the tongue and creates a zone of neutrality that determines the positioning of each tooth, the entire arch proportions, contours and alveolar process arrangement [6]. Individuals with mouth breathing condition may present an anomalous forward tongue posture to facilitate a sufficient airway space that will allow breathing [16]. The anomalous forward tongue posture produces the absence of an outward tongue pressure against the posterior arch segments. As a result the maxillary arch is squeezed inwardly by the pressure of the perioral buccinator musculature, which is unopposed by the outward tongue pressure. Then, the maxillary arch shaping grows to be distorted with a high narrow vault (Figure 3), an atypical positioning of mainly the incisor teeth, and the anterior guidance, which could determine bone and soft tissue thickness or even bone absence and the insufficient band of the attached gingiva [14,16-18]. After the midpalatal suture consolidation, there is no space for a normal posture of the tongue; then it assumes a forward posture. The anomalous forward tongue posture induces various effects. It evacuates its normal space up in the vault, determines a continuous, no physiological, and harmful contact of the tongue against the anterior teeth, with the abnormal positioning of the anterior guidance $[14,16]$. The continuous contact of the tongue against the anterior teeth and the abnormal positioning of anterior guidance may induce bone deficiency or bone loss and soft tissue thickness as well as insufficient band of attached gingiva, enhancing the accumulation of bacterial plaque, predisposing the anterior teeth to develop periodontal disease. Individuals with mouth breathing condition also present a propensity to decrease salivary flow, which may lead the anterior area to develop a periodontal disease since saliva presents antibodies as IgA and leukocytes competent to kill bacteria by phagocytosis as well as a mechanical cleaning behaviour, which assists bacteria control [19]. Treatment planning for any disease requires diagnoses of all etiologic agents that determine deleterious pathologic actions and a comprehension of how these etiologic agents may establish an association to promote tissue destruction. Then to treat any disease, it is essential to institute objective as the eradication or the precise management of all etiologic agents to the low levels, which may not induce disease activity or amplify local or general resistance against the whole etiologic factors to induce health. Open bite malocclusion is frequently not aesthetic and non-functional, and it is an etiological risk factor aiding bacteria tocause periodontal diseases. In situations of open bite malocclusion, local periodontal disease is prevalent in the anterior teeth, and to prevent anterior periodontitis it is necessary to correct the mouth breath condition and the divergence in the vertical dimension between the mandibular and maxillary anterior dental arches to eliminate or control the deleterious action which predisposes bacteria to provoke periodontal diseases. However, in some circumstances anterior open occlusal relationship in adults is difficult to treat in Brasil due to various factors such as surgical and financial conditions. Then, even without eliminating open bite malocclusion, some sequelae of periodontal diseases, such as gingival recession induced by bacteria and anterior open bite malocclusion, may be treated or made stable in the anterior region through plaque control and by enhancing the resistance of the anterior teeth by applying periodontal surgical techniques, such as improving the amount of attached $[18,19]$ keratinized gingival tissue. Nevertheless, a definition of a parameter of the quantity of the keratinized gingival tissue to improve local resistance to overcome the entire etiologic factors and treat gingival recession is always difficult to establish. Then the outcome of treatment is unpredictable. Consequently, this treatment plan may not establish health in the diseased area throughout a long stated period. Indeed, it becomes comprehensible that the major factors to be considered in treating a disease is the pthoenicity of etiologic factors against the ability of the host to resist achieving homeostasis. Then, a mouth breathing condition may provoke anterior open bite malocclusion, which when associated with opportunist bacteria may induce anterior periodontal disease.

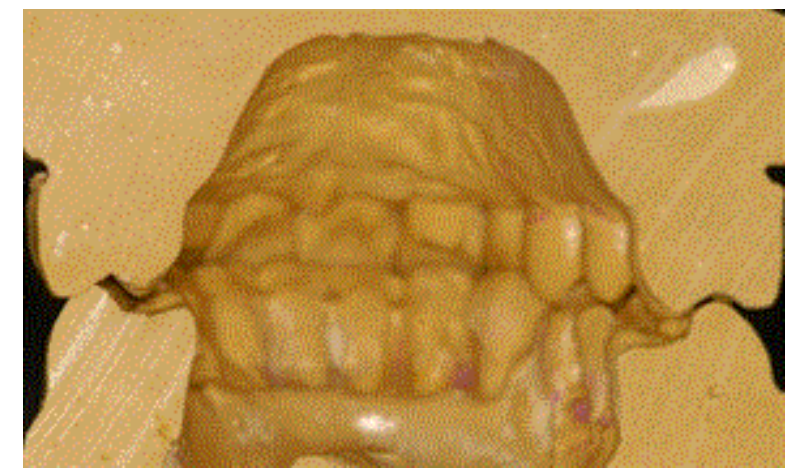

Figure 3: Maxillary arch shaping distortion with a high narrow vault.

\section{Conclusion}

Localized periodontal disease in anterior teeth is prevalent in adult individuals presenting anterior open bite relationship, a risk factor to aid bacteria to initiate periodontal disease. The precocious diagnoses and orthodontic treatment of the open bite malocclusion is essential to preventing possible development of the anterior periodontal disease.

\section{References}

1. Worms FW, Meskin LH, Isaacson RJ (1971) Open-bite. Am J Orthod 59: 589-595.

2. Cangialosi TJ (1984) Skeletal morphologic features of anterior open bite. Am J Orthod 85: 28-36.

3. Bowden BD (1966) The effects of digital and dummy sucking on arch widths, overbite, and overjet: a longitudinal study. Aust Dent J 11: 396-404. 
4. Cozza P, Baccetti T, Franchi L, Mucedero M, Polimeni A (2005 Sucking habits and facial hyperdivergency as risk factors for anterior open bite in the mixed dentition. Am J Orthod Dentofacial Orthop 128: 517-519.

5. Arat M, Iseri H (1992) Orthodontic and orthopedic approach in the treatment of skeletal open bite. Eur J Orthod 14: 207-215.

6. Arvystas MG (1977) Treatment of anterior skeletal open-bite deformity. Am J Orthod 72: 147-164.

7. Cozza P, Mucedero M, Baccetti T, Franchi L (2005) Early orthodontic treatment of skeletal open-bite malocclusion: a systematic review. Angle Orthod 75: 707-713.

8. Löe $H$, Theilade E, Jensen SB (1965) Experimental gingivitis in man. J Periodontol 36: 177-187.

9. Gibbons RJ, van Houte J (1973) On the formation of dental plaques. J Periodontol 44: 347-360.

10. Genco RJ, Löe H (1993) The role of systemic conditions and disorders in periodontal disease. Periodontol 2000 2: 98-116.

11. Koral SM, Howell TH, Jeffcoat MK (1981) Alveolar bone loss due to open interproximal contacts in periodontal disease. J Periodontol 52: 447-450.

12. Pennel BM, Keagle JG (1977) Predisposing factors in the etiology of chronic inflammatory periodontal disease. J Periodontol 48 517-532.
13. Sheiham A, Nicolau B (2005) Evaluation of social and psychological factors in periodontal disease. Periodontol 2000 39: 118-131.

14. Kina JR, Suzuki TYU, Kina EFU, Kina J, Kina M (2016) NonInflammatory destructive Periodontal Disease. Open Dent J 10: 50-57.

15. Kina JR, Suzuki TYU, Kina J, Kina M, Kina EFU (2013) Reparative phase events on periodontal disease progression: interpretation and considerations. Int J Microbiol Res 5: 439-444.

16. Dawson PE (1989) The neutral zone. In: Evaluation, diagnosis and treatment of occlusal problems ( 2 nd edn.) St. Louis, pp: 72-84.

17. Coatoam GW, Behrents RG, Bissada NF (1981) The width of keratinized gingiva during orthodontic treatment: its significance and impact on periodontal status. J Periodontol 52: 307-313.

18. Boyd RL (1978) Mucogingival considerations and their relationship to orthodontics. J Periodontol 49: 67-76.

19. Zubery Y, Machtei EE (1991) Advanced periodontal disease and development of anterior open bite. A Case Report American Journal of Dentistry 4: 295-297. 